\title{
Madarosis in acute Kawasaki disease-an uncustomary accompaniment
}

\author{
Pratap Kumar Patra ${ }^{1}$ Aaqib Zaffar Banday ${ }^{1} \cdot$ Ridhima Aggarwal $^{1} \cdot$ Ankur Kumar Jindal $^{1}$ (D) $\cdot$ Surjit Singh ${ }^{1}$
}

Received: 9 June 2021 / Revised: 1 August 2021 / Accepted: 2 August 2021 / Published online: 11 August 2021

(c) International League of Associations for Rheumatology (ILAR) 2021

Madarosis (i.e., loss of eyebrows or eyelashes) is an innocuous manifestation of a variety of systemic illnesses (for example, endocrinopathies, infections, genetic disorders) including autoimmune diseases like lupus and localized scleroderma [1]. Alopecia in acute KD, which is a rare manifestation and mostly limited to loss of scalp hair, may reflect the underlying autoimmune/inflammatory mechanisms involved in the disease pathogenesis [2-4]. However, reports of madarosis in KD are lacking. Herein, we report a novel finding of madarosis in acute $\mathrm{KD}$.

A 7-year-old girl presented with fever for 2 weeks. On examination, she had madarosis (Fig. 1), bilateral conjunctival injection, and right cervical lymphadenopathy. There was no history of contact with a suspected or proven case of SARS-CoV-2 infection. Laboratory investigations showed elevated inflammatory parameters: total white-cell count $15.2 \times 10^{9} / \mathrm{L}$ (differential: neutrophils $69 \%$, lymphocytes $24 \%$, monocytes $04 \%$, eosinophils $03 \%$ ), platelet count $640 \times 10^{9} / \mathrm{L}$ [normal $<400 \times 10^{9}$ ], erythrocyte sedimentation rate $70 \mathrm{~mm} / 1^{\text {st }}$ hour [normal $<20$ ], and C-reactive protein $52.93 \mathrm{mg} / \mathrm{L}$ [normal <6]. Serum alanine aminotransferase levels were mildly elevated $(50 \mathrm{U} / \mathrm{L}[$ normal < 40]) and mild decrease in blood hemoglobin (105 g/L [normal 110-150]) was noted. Both RT-PCR and serology for SARS-CoV-2 were negative. A diagnosis of incomplete $\mathrm{KD}$ was proffered as per the American Heart Association [2017] criteria and she was treated with intravenous immunoglobulin (IVIg, 2 $\mathrm{gm} / \mathrm{kg}$ ) and aspirin (4 mg/kg/day) [5]. Fever improved within $24 \mathrm{~h}$ of IVIg therapy. She developed periungual peeling on day 17 of her illness. Two-dimensional echocardiography remained normal throughout the disease course.

Ours is, probably, the first report of madarosis in acute KD. However, more data would be required to establish causality. Similar to alopecia in KD, autoimmunity is likely to play an important role in its evolution [3, 4]. In our case, no specific therapy for madarosis was required and improvement was noted on follow-up after IVIg therapy alone (Fig. 1). Although madarosis in KD may be disfiguring and be a source of parental concern, complete recovery in a few weeks seems to be the expected outcome.

Ankur Kumar Jindal

ankurjindal11@gmail.com

1 Allergy Immunology Unit, Department of Pediatrics, Advanced Pediatrics Centre, Post Graduate Institute of Medical Education and Research (PGIMER), Chandigarh 160012, India 
Fig. 1 Madarosis in acute Kawasaki disease. a loss of hair of both eyebrows (white arrows) and eyelashes (white arrowheads) noted at presentation; $\mathbf{b}$ closer view depicting the loss of hair of left eyebrow (white arrow) and left lower eyelash (white arrowhead); c: improvement in madarosis noted at 6 weeks of follow-up; $\mathbf{d}$ closer view depicting regrowth of hair of left eyebrow and eyelash (at 6 weeks)

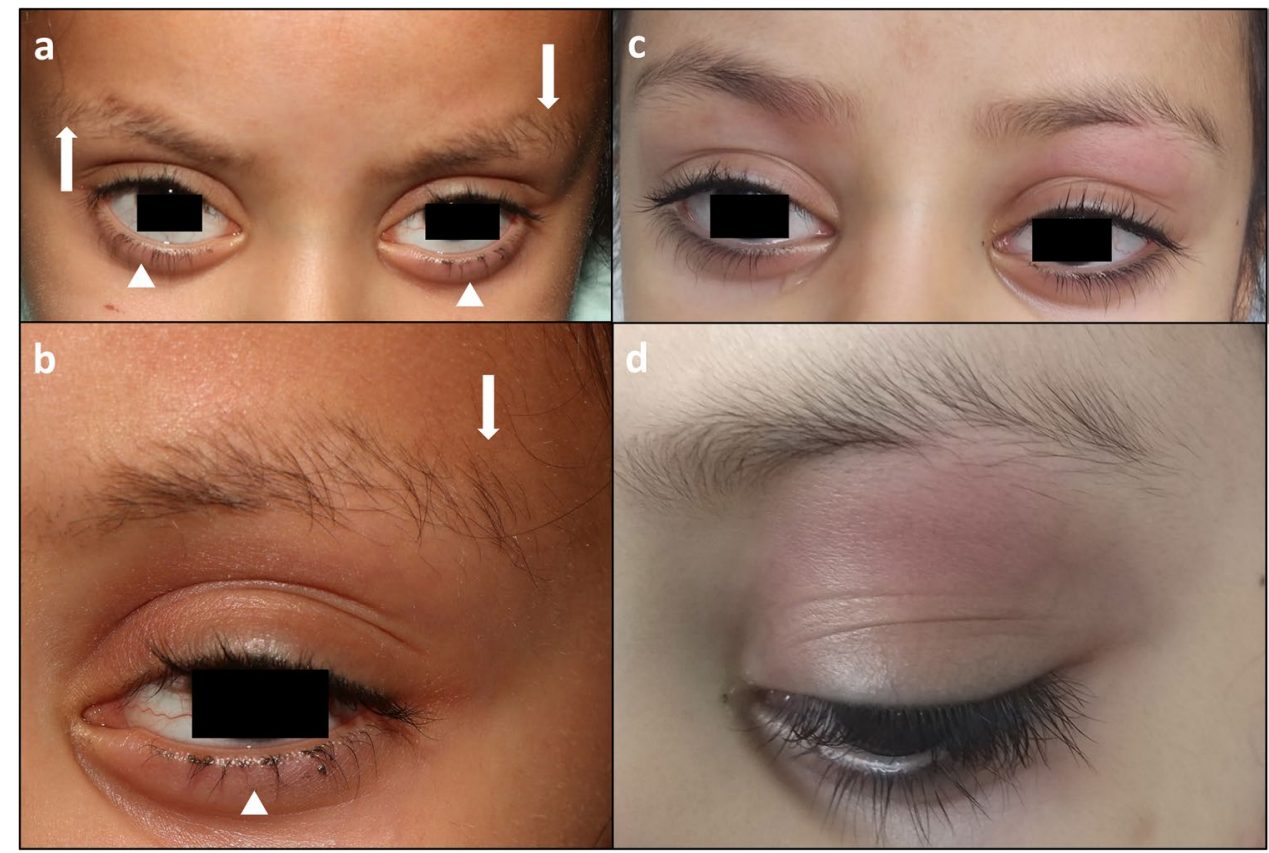

Author contributions PKP: inception of idea, writing of initial draft, editing, and revision of the manuscript, patient evaluation, management and follow-up, and review of literature. AZB: writing of initial draft, editing, and revision of the manuscript, acquisition of clinical photographs, and data collection. RA: editing and revision of the manuscript, patient follow-up, and review of literature. AKJ: editing and revision of the manuscript and patient evaluation and management. SS: editing and revision of the manuscript, patient evaluation and management, and overall supervision of manuscript preparation.

\section{Declarations}

Ethical approval and informed consent As this manuscript pertains only to a case report specific ethics approval is not mandated. Informed consent was taken from the parents of the index child before inclusion into the manuscript.

\section{Disclosure None.}

\section{References}

1. Kumar A, Karthikeyan K (2012) Madarosis: a marker of many maladies. Int J Trichology 4(1):3-18

2. Bayers S, Shulman ST, Paller AS (2013) Kawasaki disease: part I. Diagnosis, clinical features, and pathogenesis. J Am Acad Dermatol. 69(4):501.e1-11

3. Nabavizadeh SH, Safari M, Amin R (2006) Hair loss as a sign of Kawasaki disease. Iran J Allergy Asthma Immunol 5(4):199-200

4. Krishnamurthy S, Chandrashekar L, Mondal N (2012) Kawasaki disease and alopecia areata: coincidence or a true association? Pediatr Dermatol 29(4):532-534

5. Jia X, Du X, Bie S, Li X, Bao Y, Jiang M (2020) What dose of aspirin should be used in the initial treatment of Kawasaki disease? A meta-analysis. Rheumatology (Oxford) 59(8):1826-1833

Publisher's note Springer Nature remains neutral with regard to jurisdictional claims in published maps and institutional affiliations. 